\title{
Torturous path of an elastic gap band: Interdisciplinary approach to orthodontic treatment for a young patient who lost both maxillary central incisors after do-it-yourself treatment
}

\author{
Konstantonis, Dimitrios ; Brenner, Ross ; Karamolegkou, Marina ; Vasileiou, Dimitrios
}

\begin{abstract}
Spacing between the maxillary anterior teeth is a common concern for young patients and their parents. Patients often consider a diastema to be an annoying but minor problem; they might consult their general dentists for help, or try to address the issue themselves by applying elastic "gap bands." Moreover, parents, without any informed consent, sometimes accept this erroneous method as an easy and inexpensive treatment approach. A 9-year-old boy had severe acute periodontitis involving the maxillary central incisors caused by the placement of an elastic band and its apical migration. Despite periodontal and surgical interventions, the maxillary central incisors were finally extracted, and the patient started orthodontic treatment. The orthodontic treatment plan included maxillary lateral incisor substitution to replace the lost central incisors and mesialization of the maxillary posterior dentition. An interdisciplinary approach with excellent cooperation among the orthodontist, general dentist, and other dental specialists obtained an esthetically pleasing and optimized functional result. Treating the diastema between the anterior teeth with elastic gap bands and without fixed orthodontic appliances should be avoided. Patients should seek proper orthodontic advice for even small-scale orthodontic problems to prevent catastrophic outcomes, as exhibited in this case report.
\end{abstract}

DOI: https://doi.org/10.1016/j.ajodo.2018.08.009

Posted at the Zurich Open Repository and Archive, University of Zurich

ZORA URL: https://doi.org/10.5167/uzh-169491

Journal Article

Accepted Version

Originally published at:

Konstantonis, Dimitrios; Brenner, Ross; Karamolegkou, Marina; Vasileiou, Dimitrios (2018). Torturous path of an elastic gap band: Interdisciplinary approach to orthodontic treatment for a young patient who lost both maxillary central incisors after do-it-yourself treatment. American Journal of Orthodontics and Dentofacial Orthopedics, 154(6):835-847.

DOI: https://doi.org/10.1016/j.ajodo.2018.08.009 
Am J Orthod Dentofacial Orthop. 2018 Dec;154(6):835-847. doi: 10.1016/j.ajodo.2018.08.009.

\title{
Torturous path of an elastic gap band: Interdisciplinary approach to orthodontic treatment for a young patient who lost both maxillary central incisors after do-it-yourself treatment.
}

\author{
$\underline{\text { Konstantonis } \mathrm{D}^{1}}$, $\underline{\text { Brenner }}^{2}$, Karamolegkou $\mathrm{M}^{3}$, Vasileiou $\mathrm{D}^{4}$.
}

\section{Author information}

1

Department of Orthodontics, National and Kapodistrian University of Athens, Athens, Greece; Clinic for Orthodontics and Paediatric Dentistry, Center of Dental Medicine, Faculty of Medicine, 2 University of Zurich, Zurich, Switzerland. Electronic address: dikons@dent.uoa.gr.

Center for Advanced Dental Education, Department of Orthodontics, Saint Louis University, Saint Louis, Mo.

3

Department of Orthodontics, National and Kapodistrian University of Athens, Athens, Greece. 4

Private Practice, Athens, Greece.

Spacing between the maxillary anterior teeth is a common concern for young patients and their parents. Patients often consider a diastema to be an annoying but minor problem; they might consult their general dentists for help, or try to address the issue themselves by applying elastic "gap bands." Moreover, parents, without any informed consent, sometimes accept this erroneous method as an easy and inexpensive treatment approach. A 9-year-old boy had severe acute periodontitis involving the maxillary central incisors caused by the placement of an elastic band and its apical migration. Despite periodontal and surgical interventions, the maxillary central incisors were finally extracted, and the patient started orthodontic treatment. The orthodontic treatment plan included maxillary lateral incisor substitution to replace the lost central incisors and mesialization of the maxillary posterior dentition. An interdisciplinary approach with excellent cooperation among the orthodontist, general dentist, and other dental specialists obtained an esthetically pleasing and optimized functional result. Treating the diastema between the anterior teeth with elastic gap bands and without fixed orthodontic appliances should be avoided. Patients should seek proper orthodontic advice for even small-scale orthodontic problems to prevent catastrophic outcomes, as exhibited in this case report. 
Spaces in the maxillary anterior region have a variety of causes including tooth-arch and tooth-size discrepancies, abnormal frenum attachment, anteriorly proclined incisors, loss of periodontal support, and dental migration. Moreover, the diastema between the maxillary central incisors usually observed during the early mixed dentition is often spontaneously corrected with the eruption of the maxillary lateral incisors and subsequently of the maxillary canines. Parents may worry when they see such spaces in the anterior maxillary region, and seek consultation from their general dentist or family friends. Parents might be offered the "simple and inexpensive solution" of an elastic band, which the patient wears continuously or replaces daily with a new band.1, 2, 3, 4

Major adverse effects are associated with this approach, and young patients and their guardians should be aware of the risks.1, 2, 3, 4, 5 Due to the tapering anatomy of the central incisor root, the elastic band can easily slip toward the neck of the tooth and move apically along the periodontal ligament on its path to the tooth's apex. This adverse effect is often irreversible and causes significant bone loss that jeopardizes the tooth's stability and prognosis. Experimental studies in animal models have shown that placing an elastic band around a tooth can cause acute periodontitis.6, 7, 8

Diagnosis and etiology

A boy, age 8 years 2 months, was brought to the Pediatric Dentistry Department of the Dental School of the National and Kapodistrian University of Athens in Greece complaining of pain and swelling around his maxillary central incisors. The clinical examination showed that the maxillary central incisors were extruded; they exhibited grade 2 mobility. The parents mentioned that they had used an elastic gap band to close the gap between the central incisors. They said that the rubber band was visible for 2 days, and then they could not see it anymore. They believed that the elastic band had most likely broken but were unconcerned since the gap was successfully eliminated, and everybody was happy with this do-it-yourself treatment. During the consultation at the pediatric dentistry department, a passive 0.018-in Australian archwire was bonded onto the labial surfaces of the maxillary central incisors and deciduous canines to stabilize the mobile teeth (Fig 1). The patient was referred to the Department of Periodontology for further evaluation. 

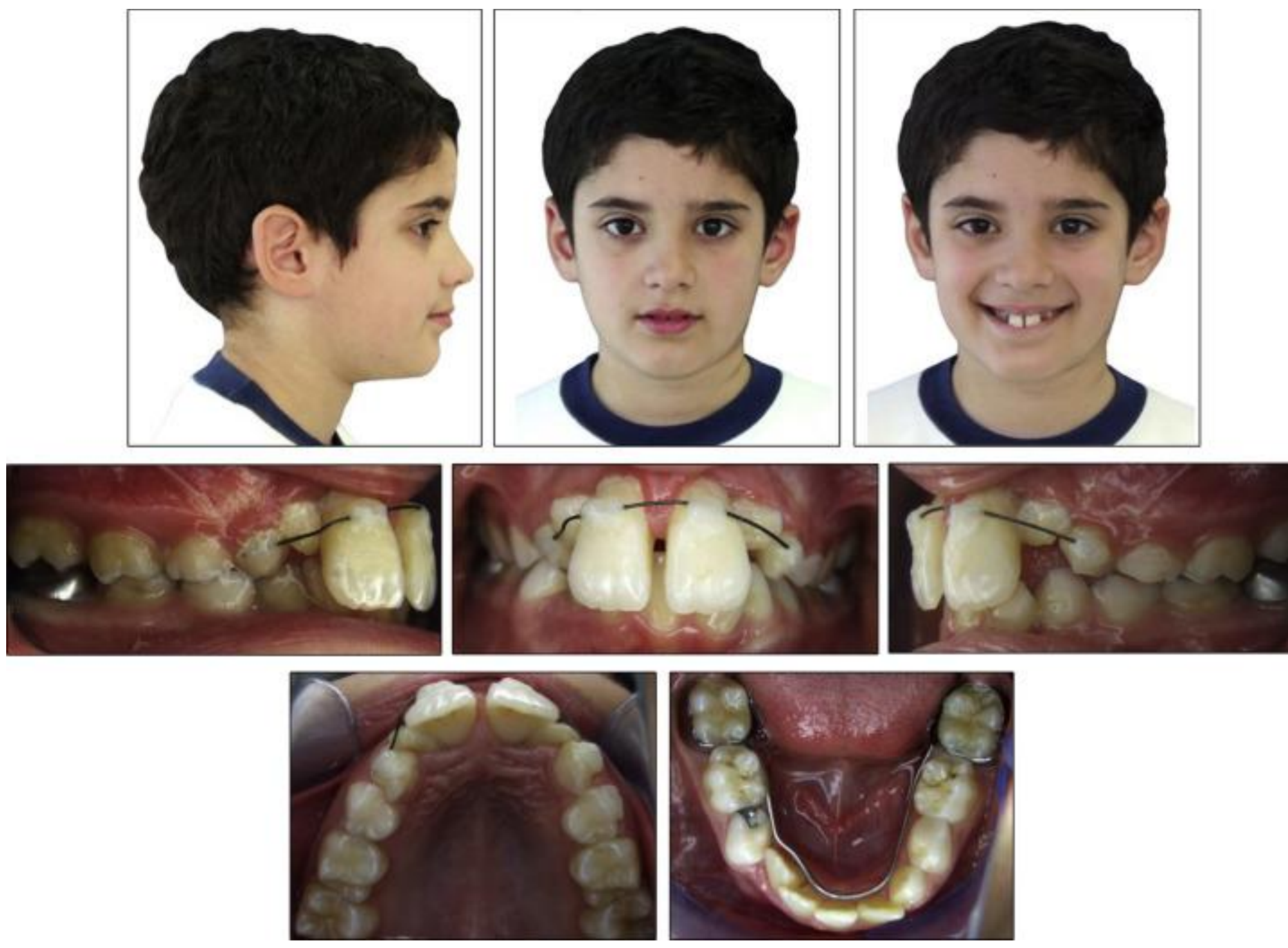

Fig 1. Initial facial and intraoral photographs.

The periodontal examination of the maxillary central incisors confirmed grade 2 mobility with pocket depth measurements of $10 \mathrm{~mm}$. The radiographic evaluation consisted of panoramic and periapical $\mathrm{x}$ rays of the maxillary anterior region (Fig 2). Vertical bone loss was shown radiographically, and the teeth were estimated to have lost more than $75 \%$ of their bone support. The periodontal diagnosis was acute severe periodontitis for an unknown reason. The rest of the dentition had no periodontal problems. The patient was seen every week, and the mobility of the incisors continued to worsen. Three weeks after the initial visit, it was decided to intervene surgically. Under local anesthesia, the periodontist performed open flap debridement with deep scaling of the roots. An elastic band around the apices of the 2 central incisors was found and removed. Emdogain was applied along the root surfaces to enhance bone turnover, ostein regeneration, and periodontal ligament reattachment. The flaps were sutured, and the patient was administered amoxicillin for a week (Fig 3). A new passive wire was bonded to stabilize the maxillary central incisors, and the patient was referred to the Department of Orthodontics. 

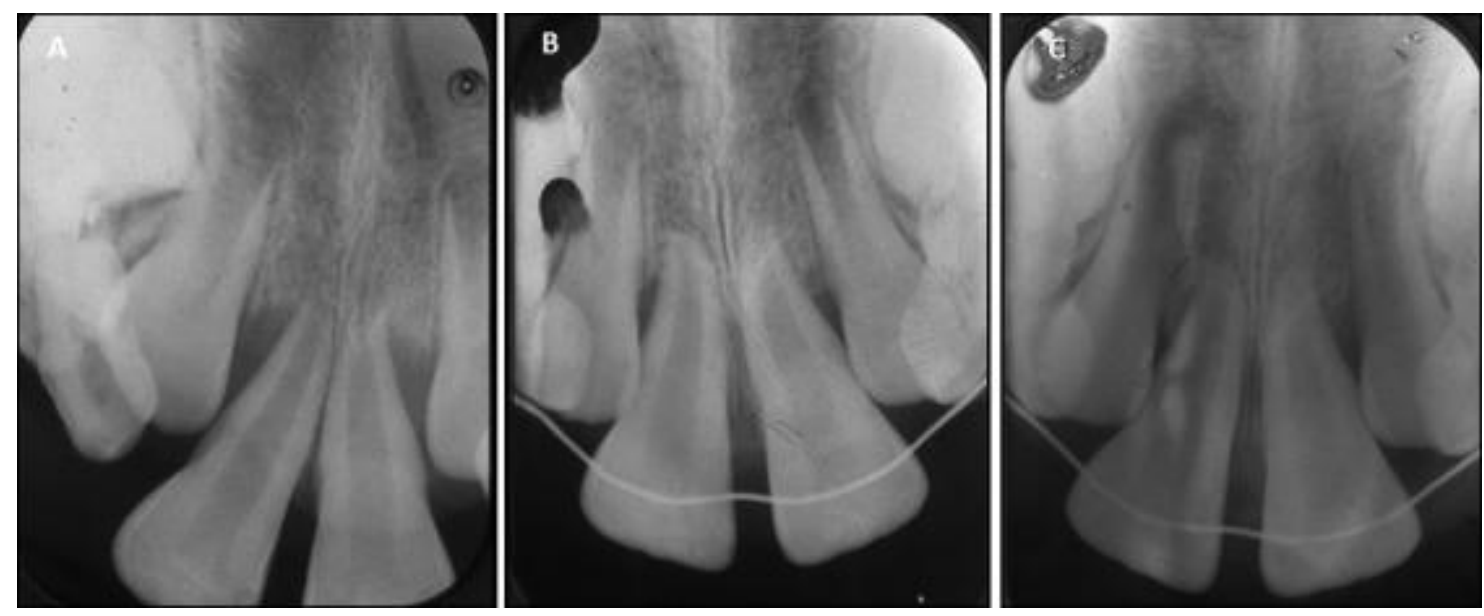

Fig 2. Periapical radiographs: A, start of treatment; B, 3 months after placement of stabilizing wire; C, 4 months after placement of stabilizing wire.
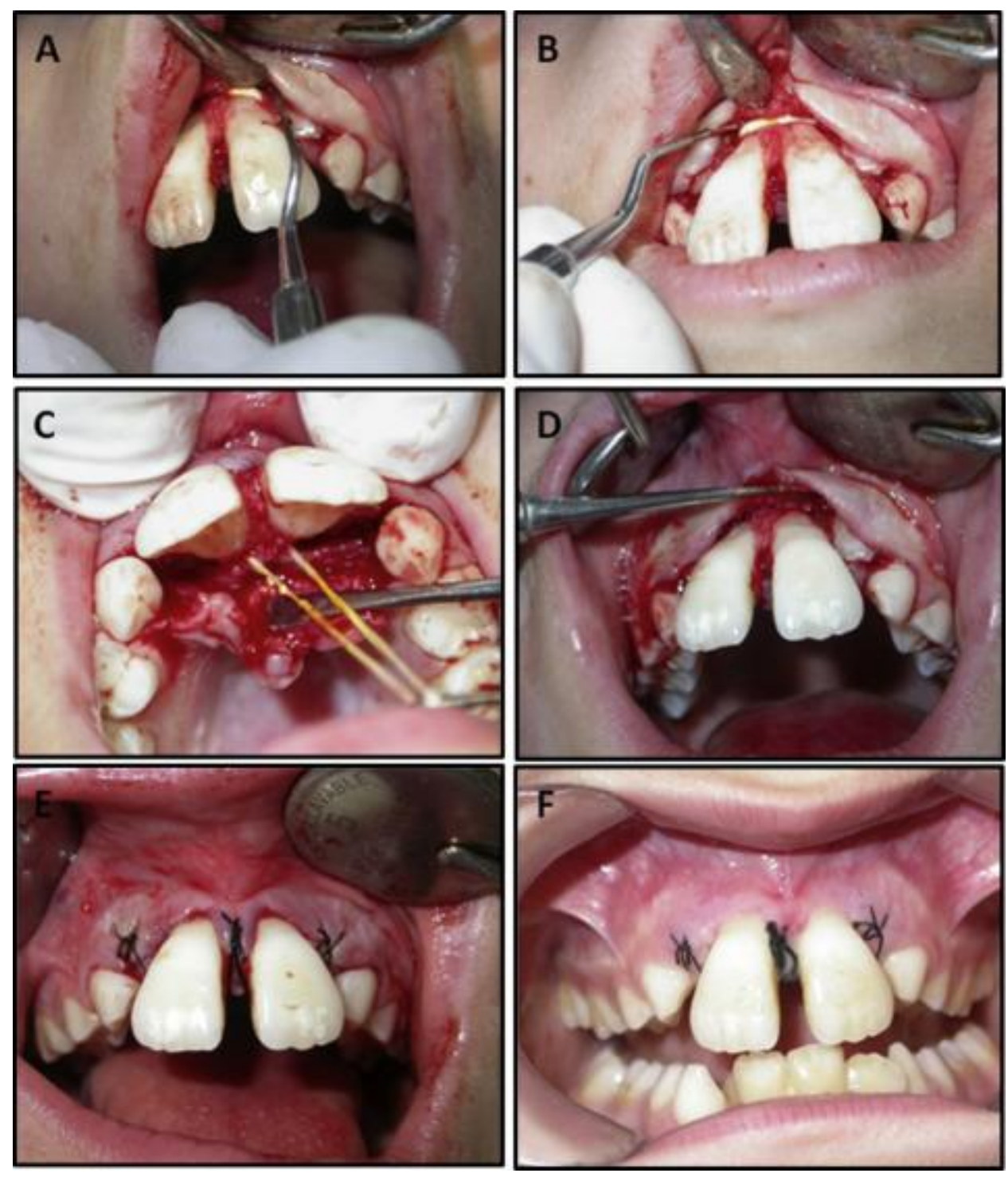
Fig 3. A-D, Open flap debridement with deep scaling of the roots showed an elastic band around the apices of the 2 central incisors; $E$, the elastic band was removed, and the flaps were sutured; F, 1 week after surgery.

Upon orthodontic screening, the facial evaluation showed increased lower face height with a convex soft tissue profile, and lip incompetence in a relaxed position. The intraoral examination showed that the patient was in the intertransitional phase of the mixed dentition with a Class I molar relationship on the left side and a Class II molar relationship on the right side. Overjet was $3.5 \mathrm{~mm}$, overbite was $7 \mathrm{~mm}$, and the mandibular midline was deviated $3.5 \mathrm{~mm}$ to the right relative to the facial midline (Figs 1 and 4). Also, according to the Moyers analysis, the patient had $1.2 \mathrm{~mm}$ of crowding on the maxillary left side and $0.3 \mathrm{~mm}$ of spacing on the right side, resulting in a total of $0.9 \mathrm{~mm}$ of crowding in the maxillary arch. In the mandibular arch, the tooth-arch discrepancy included $5.4 \mathrm{~mm}$ of crowding in the right quadrant and $1.5 \mathrm{~mm}$ of excess space in the left quadrant, resulting in a total of $3.9 \mathrm{~mm}$ of mandibular crowding. To maintain the leeway space, a mandibular lingual holding arch had been previously placed by the pediatric dentist.
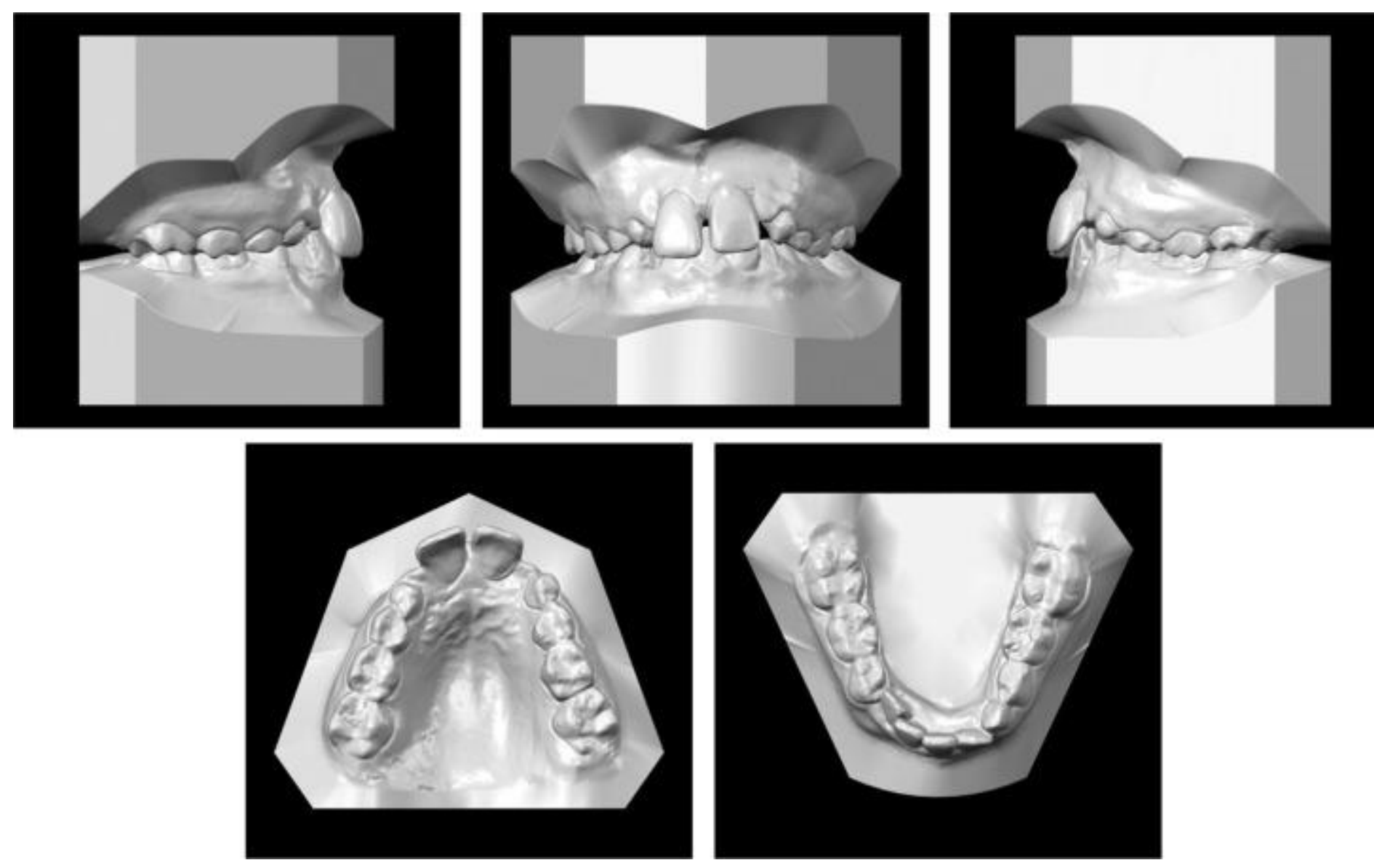

Fig 4. Initial digital dental casts.

The cephalometric analysis (Fig 5; Table) showed a skeletal Class I relationship (ANB angle, $3.3^{\circ}$ ) with a normally positioned maxilla and a slightly retrusive mandible. The patient had no further skeletal 
discrepancies, and the positions of the maxillary and mandibular incisors as well as the interincisal angle were within normal limits. His smile was unattractive because of the extruded maxillary central incisors, and mentalis muscle strain was observed upon lip closure.

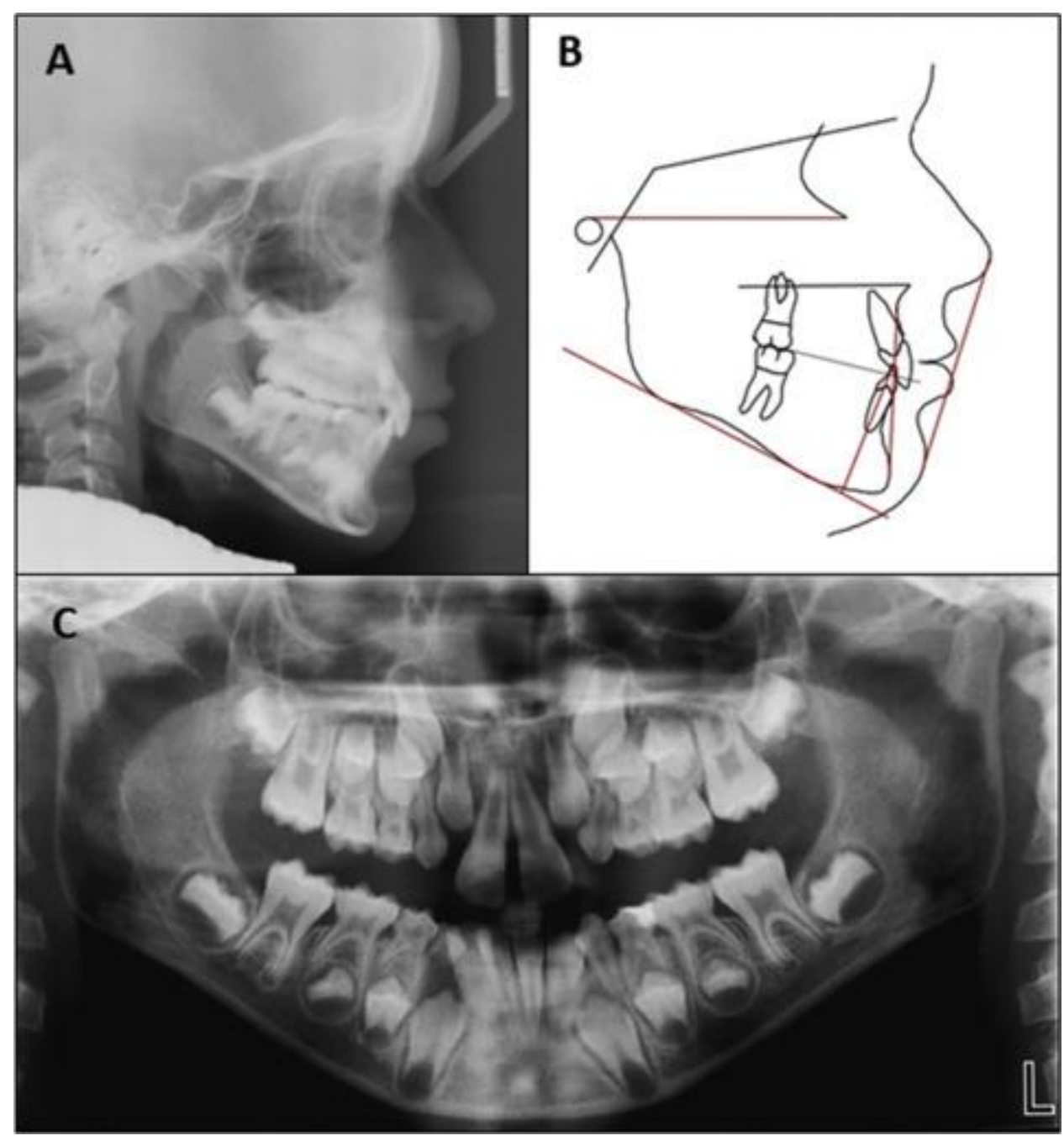

Fig 5. A, Initial lateral cephalometric radiograph; B, tracing; C, panoramic radiograph.

Table. Cephalometric measurements

Pretreatment

Norm

Posttreatment

Skeletal

SNA $\left({ }^{\circ}\right)$

78.4

82

77.6

SNB $\left({ }^{\circ}\right)$

75.5

80

75.2

ANB $\left({ }^{\circ}\right)$

2.8

2.4 


\begin{tabular}{|c|c|c|c|}
\hline & Pretreatment & Norm & Posttreatment \\
\hline SNPog $\left({ }^{\circ}\right)$ & 77.1 & 81 & 77.8 \\
\hline $\operatorname{FMA}\left({ }^{\circ}\right)$ & 27.4 & 25 & 26.5 \\
\hline SN-PP $\left({ }^{\circ}\right)$ & 10.9 & 8.5 & 13.2 \\
\hline$y$-axis $\left({ }^{\circ}\right)$ & 54.4 & 59 & 53.9 \\
\hline UFH/TFH (mm) & 44.4 & 44 & 45.6 \\
\hline LFH/TFH (\%) & 55.6 & 56 & 54.4 \\
\hline GoGn-SN $\left({ }^{\circ}\right)$ & 35.6 & 32 & 34.1 \\
\hline \multicolumn{4}{|l|}{ Dental } \\
\hline U1-FH & 109.7 & 110 & 119 \\
\hline U1-SN ( $\left.{ }^{\circ}\right)$ & 107 & 110 & 97,9 \\
\hline U1-PP $\left({ }^{\circ}\right)$ & 108.8 & 110 & 120.3 \\
\hline U1-APog $\left({ }^{\circ}\right)$ & 22.2 & 22 & 29 \\
\hline U1-APog (mm) & 4.1 & 2.5 & 5.2 \\
\hline $\operatorname{IMPA}\left({ }^{\circ}\right)$ & 84.6 & 90 & 88.7 \\
\hline L1-APog $\left({ }^{\circ}\right)$ & 19.5 & 23 & 25.2 \\
\hline L1-APog (mm) & -0.2 & 0 & 1.8 \\
\hline Interincisal angle $\left({ }^{\circ}\right)$ & 138.3 & 135 & 125.7 \\
\hline \multicolumn{4}{|l|}{ Soft-tissue } \\
\hline Nasiolabial angle $\left({ }^{\circ}\right)$ & 110 & 105 & 103.5 \\
\hline Upper lip to E-plane (mm) & -1.7 & 0 & -3.7 \\
\hline Lower lip to E-plane (mm) & 0.8 & 1 & -2.5 \\
\hline Z-angle $\left({ }^{\circ}\right)$ & 74.8 & 78 & 73.8 \\
\hline
\end{tabular}

Treatment alternatives 
Two treatment options were presented to address the orthodontic problems and the potential loss of the maxillary central incisors. Both options included extraction of the 2 maxillary central incisors. The first option aimed at prosthetic replacement of the lost incisors with a Maryland-type bridge, which would be replaced by 2 implants after growth had ceased. Alternatively, the second option involved lateral incisor substitution for the maxillary central incisors followed by esthetic composite buildups or prosthetic restorations. In this option, all posterior teeth would be moved mesially, with the canines and first premolars receiving appropriate restorations for esthetics. The patient's parents decided to move forward with the second option.

\section{Treatment objectives}

The treatment goal for this young patient was to address the loss of the maxillary central incisors by moving the lateral incisors into the central incisors' area, and the canines into the lateral incisors' area, achieving a functional occlusal scheme. Therefore, at the end of treatment, the first molars should occlude in a Class II molar relationship, with the canines in a Class I relationship with substitution of the maxillary canines by the first premolars. Our treatment objectives were to maintain or improve the skeletal plane angles and lower face height to obtain ideal overbite and overjet and to correct the mandibular dental midline, thus providing a stable, functional occlusion complemented by a satisfactory soft-tissue profile. Additionally, an interdisciplinary approach involving the general dentist, periodontist, and prosthodontist would be implemented.

\section{Treatment progress}

It was decided to proceed with a slow forced eruption of the central incisors to maintain the integrity and height of the supporting alveolar bone. As the central incisors were moved occlusally, their incisal edges were trimmed to an equal amount of the "step down" bends placed in the archwire (Figs 6 and 7). During the forced eruption phase, the patient wore an anterior biteplate to prevent premature anterior contacts.

Fig 6. Intraoral progress photographs of the forced eruption process: A, start of treatment; B, start of treatment with the patient wearing a biteplate appliance; $C, 10$ months after forced eruption began; $D$, 12 months after forced eruption began.
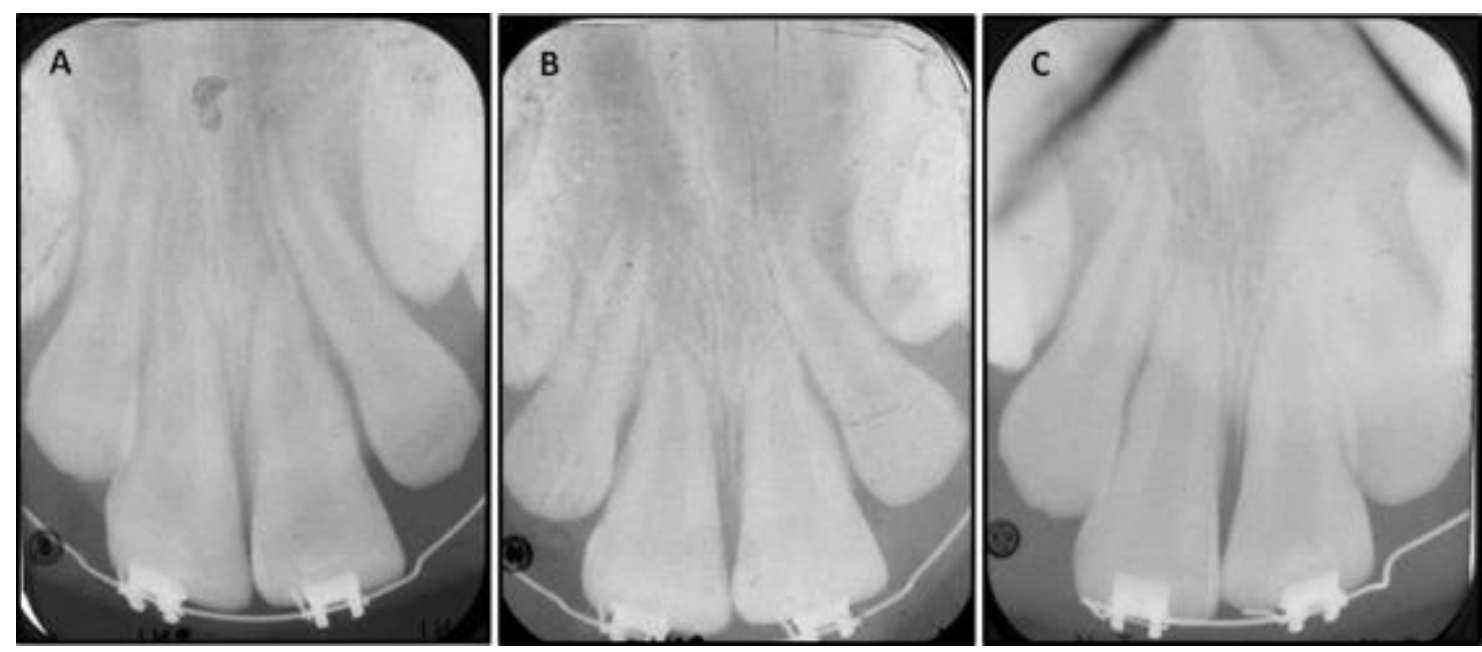

Fig 7. Periapical radiographs during the forced eruption process: $A$, start of forced eruption; $B, 6$ months after forced eruption began; $C, 10$ months after forced eruption began.

Forced eruption of the central incisors proceeded slowly during the first 12 months of treatment until they were finally extracted. Composite buildups were placed on the maxillary lateral incisors followed by full bonding of both dental arches with standard edgewise appliances with 0.022-in slots. The archwire sequence during levelling was 0.014-in nickel-titanium, 0.016-in nickel-titanium, and 0.018-in Australian wires in both arches.

Then, a $0.016 \times 0.022$-in stainless steel archwire was used while moving the lateral incisors into the position of the central incisors, allowing the canines to erupt mesially. When the maxillary canines erupted, their cusps were slightly trimmed to start resembling lateral incisors. Then, a $0.017 \times 0.025$-in stainless steel archwire was selected to provide stability during the mesialization of the canines and first premolars via sliding mechanics (Fig 8). Furthermore, open-coil nickel-titanium springs were used from the maxillary molars to the second premolars with Class III elastic anchorage and then removed once the second premolar was in its appropriate position (Fig 9). Finally, Class III elastics were used to achieve mesial displacement of the maxillary first and second molars. 




Fig 8. Panoramic radiograph, 2 years 10 months into treatment.
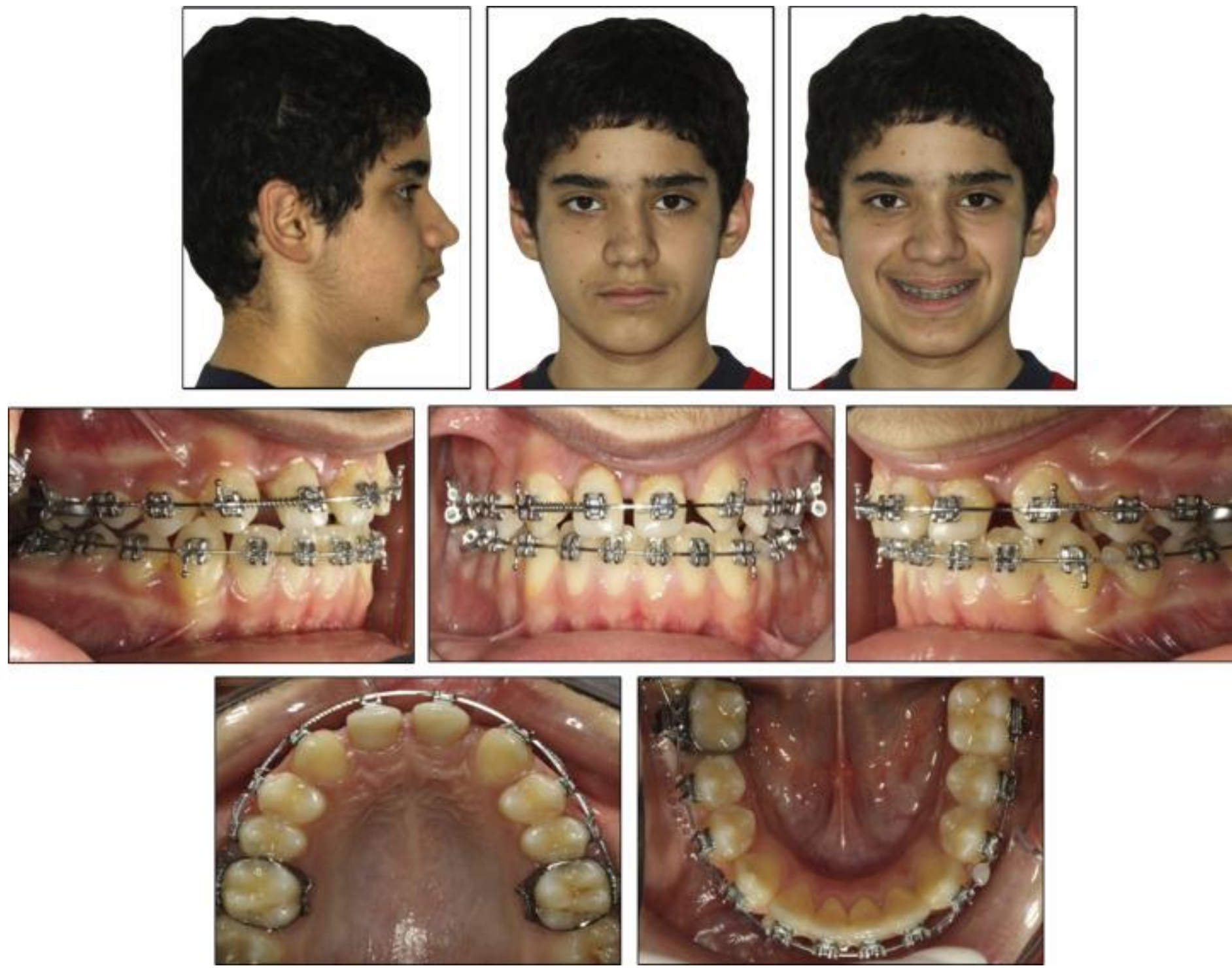
Fig 9. Facial and intraoral photographs 3 years into treatment.

The composite restorations of the maxillary lateral incisors were completed during treatment to achieve the final mesiodistal widths of the teeth before finishing mechanics. Lingual fixed retainers of 0.0175 -in multistranded stainless steel wires were placed on the maxillary incisors and the mandibular incisors and canines. Additionally, Hawley wrap-around retainers were delivered for both arches.

Treatment results

Both arches were leveled and aligned, and a functional occlusion with Class II molar and Class I canine relationships with canine substitution by the first premolars was achieved (Fig 10). The final overjet and overbite were ideal (Fig 11), and the final panoramic radiograph showed good root parallelism (Fig 12). The alveolar bone height in the anterior area was increased by the treatment. The superimposition of the initial and final cephalometric radiographs (Fig 13) showed that growth was favorable, whereas adequate vertical control was achieved during the orthodontic treatment. The maxillary and mandibular incisors were properly positioned over their basal bones and in relation to the face, and lip positions in relation to the E-plane were esthetically pleasant. The treatment duration was 3 years 9 months. The 5 year posttreatment records showed that the results remained stable. The alveolar bone height in the anterior area was maintained, and the outcome satisfied both esthetic and functional demands (Figs 14 and 15). 

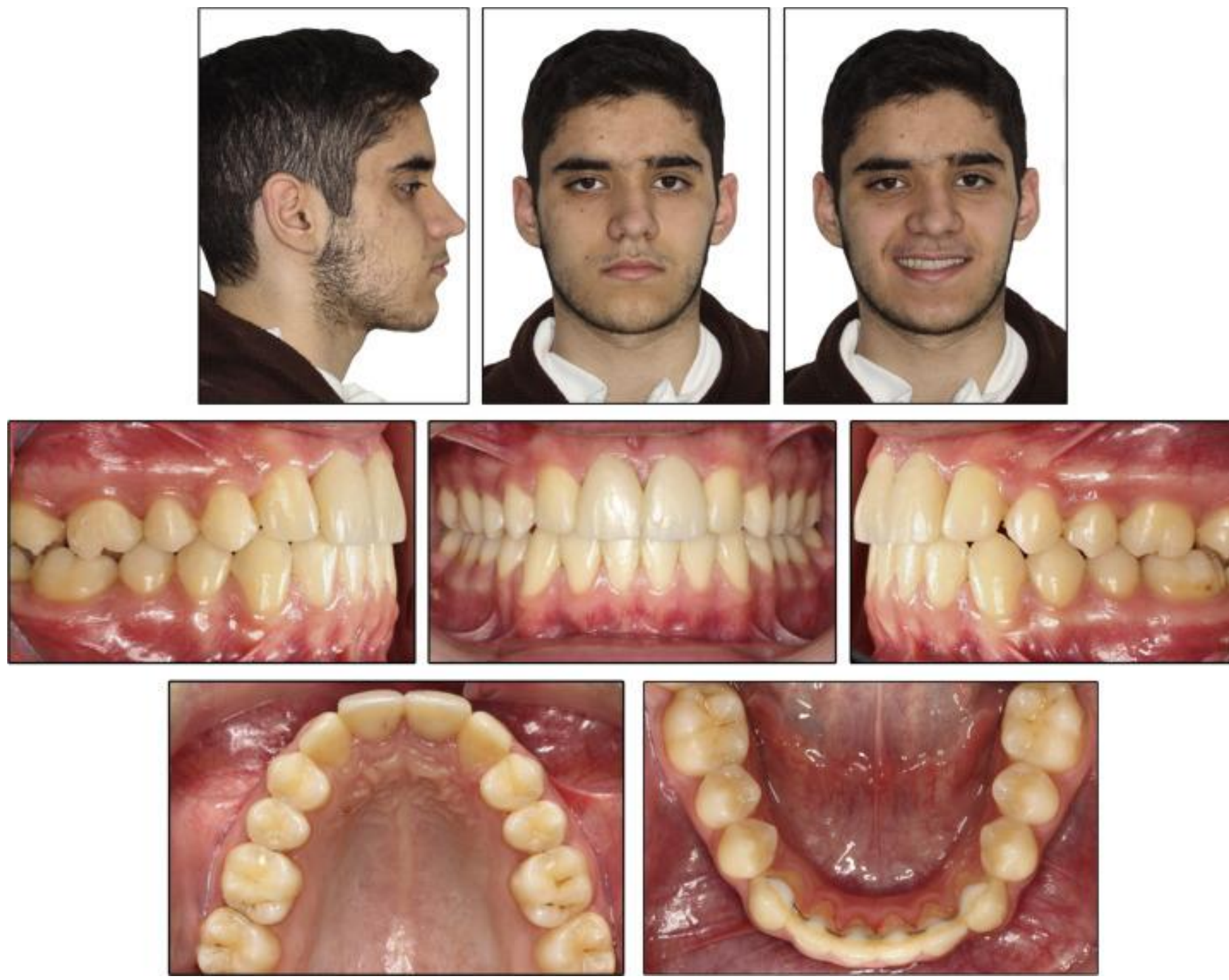

Fig 10. Final facial and intraoral photographs. 

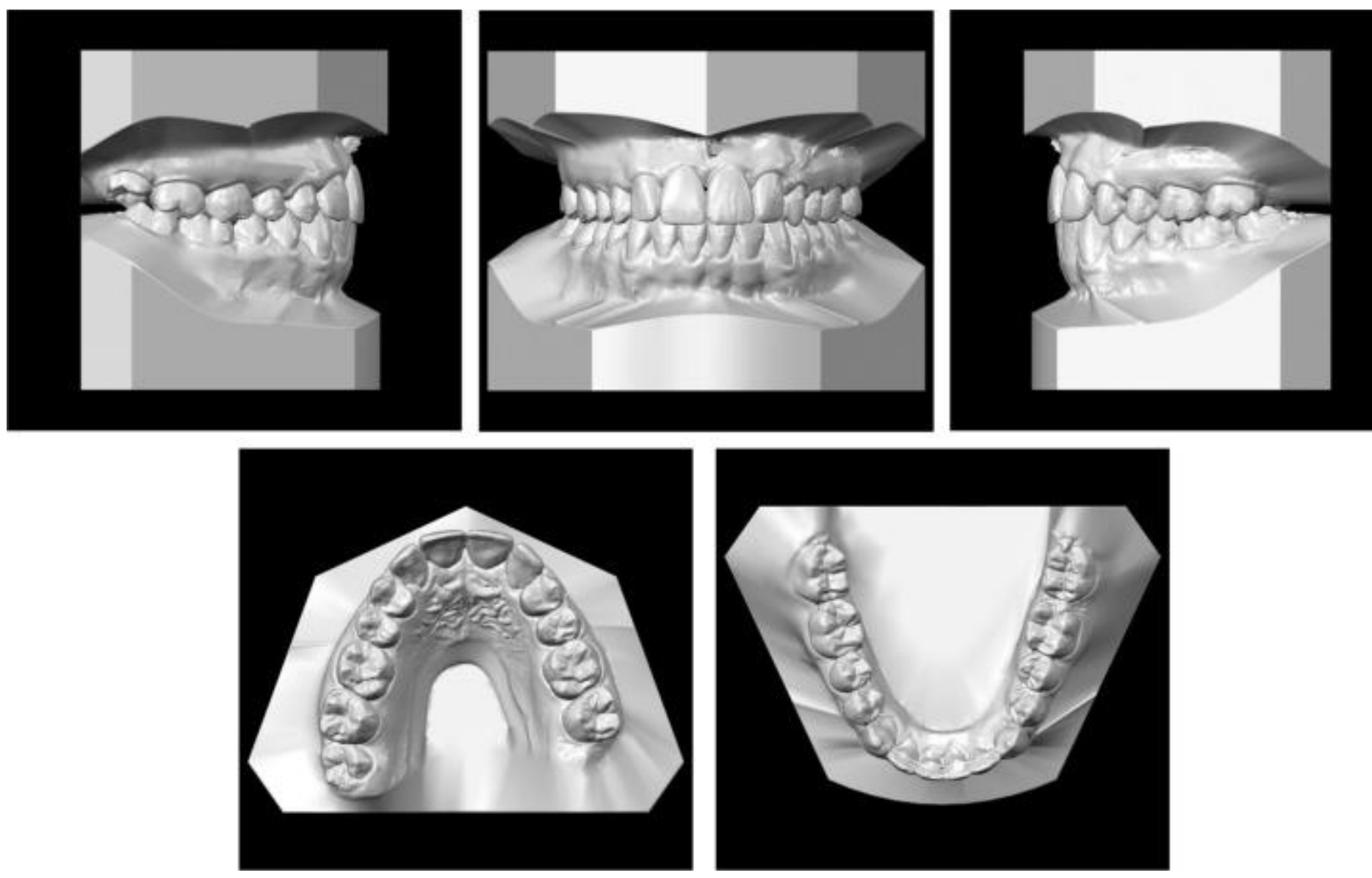

Fig 11. Final digital dental casts. 


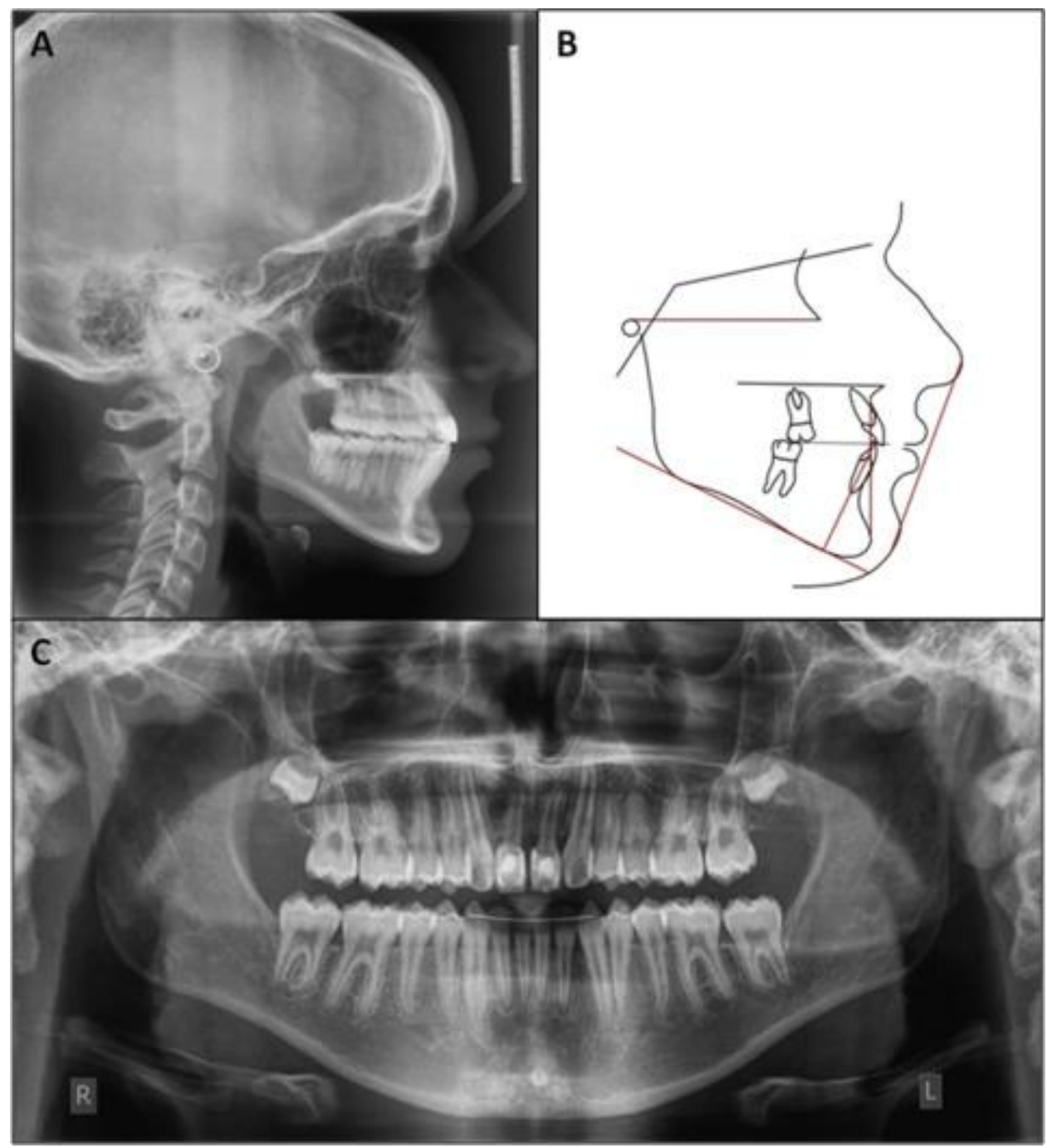

Fig 12. A, Final lateral cephalometric radiograph; B, tracing; C, panoramic radiograph.

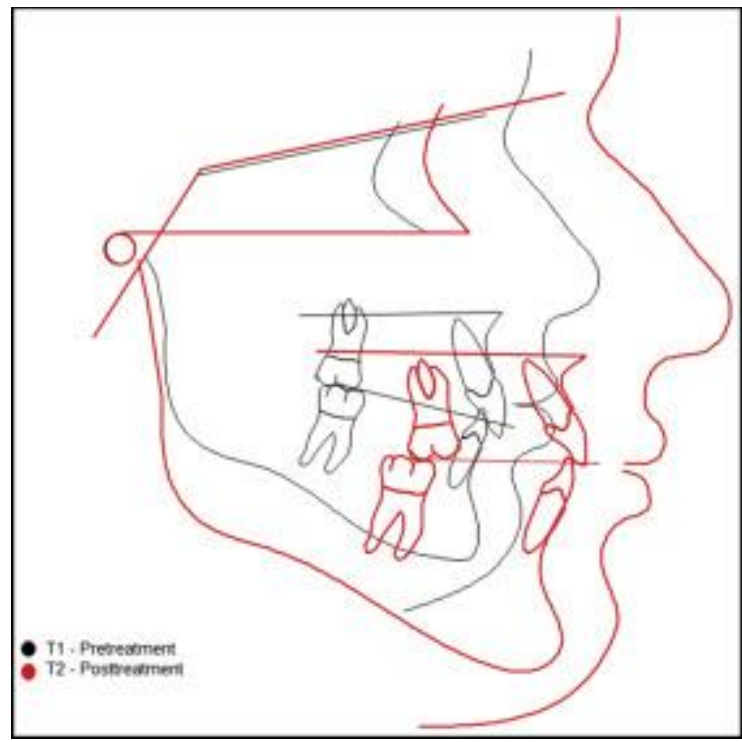


Fig 13. Superimposition of initial and final cephalometric tracings.
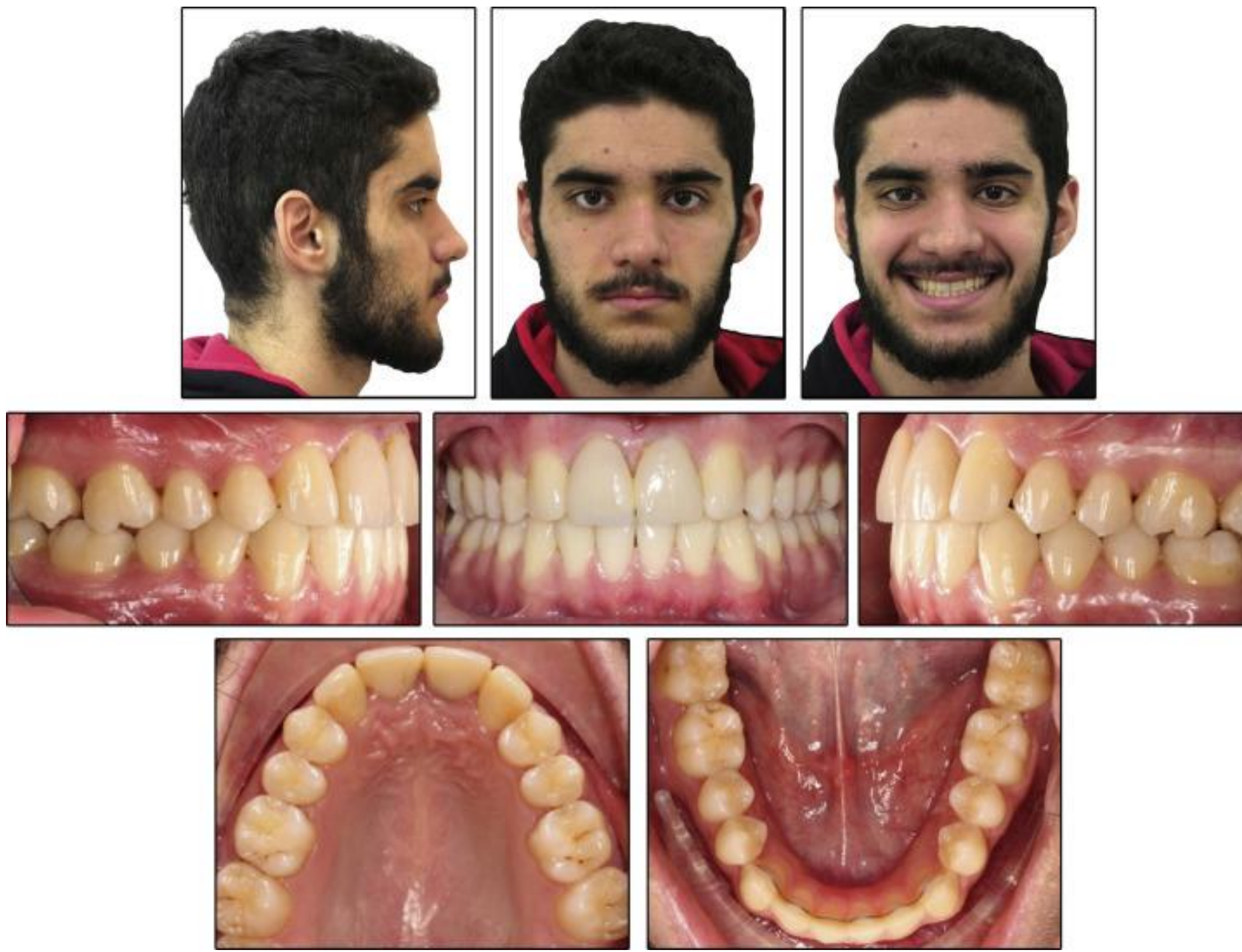

Fig 14. Five-year retention facial and intraoral photographs. 


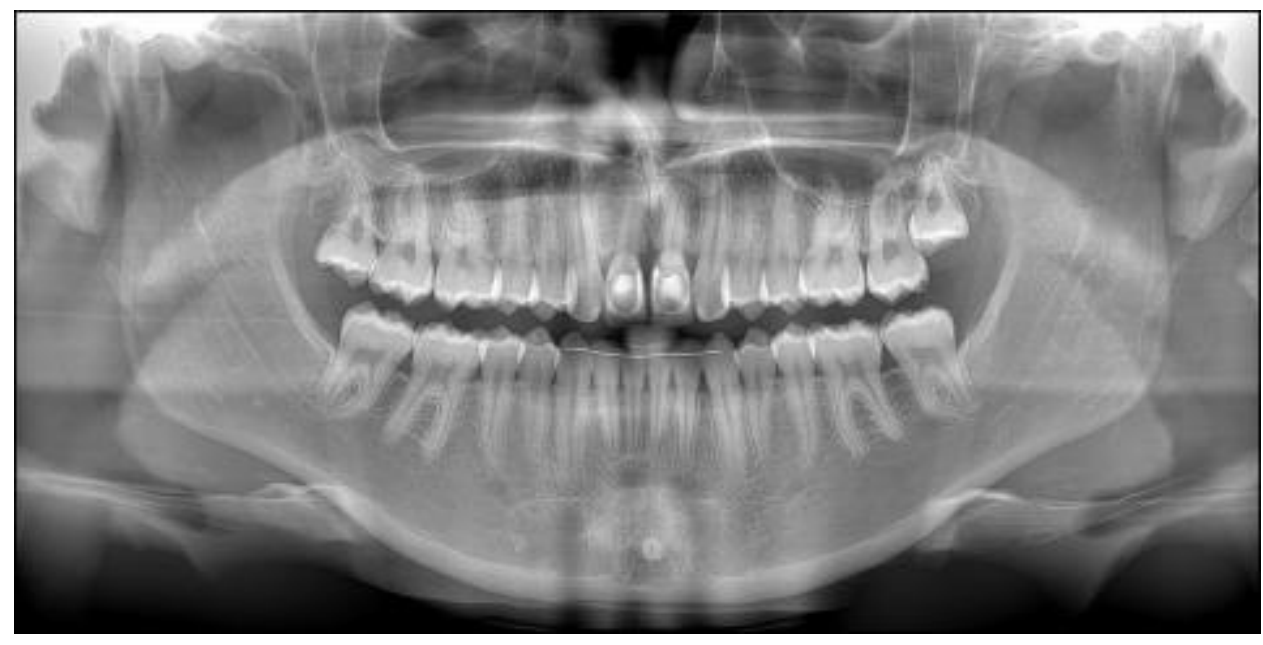

Fig 15. Five-year retention panoramic radiograph.

\section{Discussion}

The use of elastic gap bands in an attempt to close a diastema between the maxillary incisors is quite common.9 Nevertheless, the harmful effects of this approach have been reported in numerous cases since 1870.10 Lack of proper information regarding the dangers that these gap bands may cause to the teeth they are placed on represents the latest unsafe trend of self-treating: a trend that has made consumers consider possible do-it-yourself options for closing the central incisor diastema.1

In this patient, placement of the elastic band on the maxillary central incisors caused severe periodontal damage that led to their loss and made replacement a complex procedure requiring an interdisciplinary approach.2, 3, 4, 5, 11, 12, 13, 14, 15 Huang and Creath16 concluded in their literature review that elastics should not be used around teeth without stabilization through brackets or directly bonded to the teeth. The effect of these elastic bands on the periodontal tissues is well known, since they have been used when atraumatic extractions of teeth are needed, such as in bisphosphonate-treated patients or in patients with hemophilia.17, 18

Moreover, in patients similar to ours, early diagnosis and intervention are absolutely essential to prevent rapid decay in the prognosis of the teeth. In similar conditions of localized acute periodontitis in an otherwise healthy, dentally and periodontally stable patient, the clinician should be suspicious about foreign bodies in the alveolus, and a detailed radiographic examination should be requested. It is recommended that all orthodontic elastics should have radiopaque materials incorporated into them to aid in their radiographic localization. Then, in these patients, periodontal exploration through surgical intervention may be carried out in an expedient manner to improve the prognosis of the affected teeth.

In our patient, the prognosis was hopeless for the teeth involved, and forced eruption of the maxillary central incisors before their extraction improved bone density and stimulated local alveolar bone regeneration. Orthodontic extrusion has been suggested by many clinicians as a technique for pocket depth reduction and increasing bone density in patients with severe bone loss.19, 20, 21, 22, 23 The decision to mesialize the maxillary lateral incisors into the positions of the central incisors and, 
subsequently, all posterior teeth, was made to promote alveolar ridge development and prevent future prosthetic replacement.

In patients with congenitally missing lateral incisors, canine substitution for the lateral incisors is a relatively common technique with satisfactory long-term results. Space closure as a treatment alternative has been discussed in many publications.24, 25, 26, 27, 28, 29, 30 Czochrowska et al26 concluded that orthodontic mesialization of the lateral incisor to replace a missing central incisor is a valid treatment modality with appropriate attention to detail. Robertsson and Mohlin27 concluded, in their long-term study, that functional and periodontal evaluation as well as patients' satisfaction were better in space closure than in the prosthetic approach. One main advantage of this technique is that treatment can be finished in early adolescence, with the teeth and periodontal structures appearing natural many years after treatment.24

To achieve an esthetic result in this patient, restorative interventions were needed. Cooperation between various specialties is vital for orthodontic-restorative patients.25, 28 The functional evaluation showed that canine substitution results in a different occlusal pattern, as in group function. Nordquist and McNeill30 reported no significant difference in occlusion and periodontal health between canine guidance and group function.

On the other hand, implant placement instead of space closure leads to a Class I molar and canine relationship with a mutually protected occlusal pattern. Even though implant use can lead to an esthetic result in the short term, there are many considerations for long-term results. There is evidence in many studies that implants in the anterior region, especially in young adults, tend to be infraoccluded over time, and significant bone loss involving the teeth adjacent to the implants has been noted.31, 32, 33, 34, 35, 36, 37 Rosa and Zachrisson34 reported progressive infraocclusion, even when the implants were placed in mature adults, lack of uprighting compared with natural incisors, blue color of the labial gingiva, visibility of metal or porcelain abutment over time, interdental recession (regarding particularly the distal papilla), and difficulty of achieving a natural-looking porcelain crown.

Similar concerns about the esthetic problems of implants in the anterior zone have been reported in other publications.35, 36, 38, 39 Silveira et al31 reported in a systematic review that periodontal scores were better with space closure than with prostheses. Also, in their review, the prosthetic replacement received a worse esthetic evaluation than did space closure. Furthermore, orthodontists should also be aware of the long-term survival of implants. Jung et al40 and Scurria et al41 reported, in their systematic reviews, important percentages of prosthetic restoration losses due to complications with an implant's parts or with the prosthetic restorations on them.

Considering these disadvantages and our patient's age, a treatment plan with implants would not have been the treatment of choice for him. Moreover, bone loss due to the acute, localized periodontal disease was so excessive that the safe placement of the implant would have been compromised. Then bony reconstruction would have been necessary and still mght not have guaranteed optimum implant esthetics.42 Of course, due to the tendency for diastema opening in patients treated with space closure, the use of a fixed lingual retainer was considered necessary. 43 


\section{Conclusions}

This case report presents the interdisciplinary approach to orthodontic treatment of a young boy who lost his maxillary central incisors after the placement of an elastic gap band. Clinicians and patients should be aware that the inappropriate use of elastic bands in addressing diastemata may result in severe periodontal destruction and eventual tooth loss. When the decision for space closure is made to achieve an esthetic and functional result, an interdisciplinary treatment approach is considered indispensable.

\section{References}

1 M.G. Alves, D. Kitakawa, J.B. Becker, A.A. Brandão, L.A. Cabral, J.D. AlmeidaElastic band causing exfoliation of the upper permanent central incisors Case Rep Dent, 2015 (2015), p. 186945

2 M.N. Al-QutubOrthodontic elastic band-induced periodontitis-a case report Saudi Dent J, 24 (2012), pp. $49-53$

3 J.E. AdcockExfoliation of maxillary central incisors due to misapplication of orthodontic rubber bands Tex Dent J, 116 (1999), pp. 8-13

4 C.B. Olsen, A.W. PollardSevere bone loss caused by orthodontic rubber bands; management and nineyear follow-up: report of case ASDC J Dent Child, 65 (1998), pp. 25-28

5 M. Redlich, E.A. Galun, Y. ZilbermanOrthodontic-prosthetic treatment to replace maxillary incisors exfoliated because of improper use of orthodontic elastics: a case report Quintessence Int, 28 (1997), pp. $241-244$

6 H.E. Schroeder, J. LindheConversion of stable established gingivitis in the dog into destructive periodontitis Arch Oral Biol, 20 (1975), pp. 775-782

7 J.G. Caton, H.A. ZanderPrimate model for testing periodontal treatment procedures: I. Histologic investigation of localized periodontal pockets produced by orthodontic elastics J Periodontol, 46 (1975), pp. 71-77

8 C.M. Waldo, J.M. RothblattHistologic response to tooth movement in the laboratory rat; procedure and preliminary observations J Dent Res, 33 (1954), pp. 481-486

9 R.G. BehrentsConsumer alert on the use of elastics as "gap bands." Am J Orthod Dentofacial Orthop, 146 (2014), pp. 271-272

10J.H. McQuillenCare in the use of Indian rubber rings (editorial)Dent Cosmos, 12 (1870), p. 429

11S. Rafiuddin, P.K. Yg, S. Biswas, S.S. Prabhu, C. Bm, R. Mplatrogenic damage to the periodontium caused by orthodontic treatment procedures: an overviewOpen Dent J, 9 (2015), pp. 228-234 
12Y.T. Lin, Y.L. Huang, S.H. Chang, H.H. HongSequelae of iatrogenic periodontal destruction associated with elastics and permanent incisors: literature review and report of 3 cases Pediatr Dent, 33 (2011), pp. 516-521

13G. St George, M.A. DonachieCase report: orthodontic separators as periodontal ligatures in periodontal bone lossEur J Prosthodont Restor Dent, 10 (2002), pp. 97-99

14W.L. Pan, C.P. Chan, C.P. SuLocalized periodontitis induced by rubber bands. Report of two cases Changgeng Yi Xue Za Zhi, 14 (1991), pp. 54-60

15V.A. Marino, H.R. Fry, R.G. BehrentsSevere localized destruction of the periodontium secondary to subgingival displacement of an elastic bandJ Periodontol, 59 (1988), pp. 472-477

16W.J. Huang, C.J. CreathThe midline diastema: a review of its etiology and treatment Pediatr Dent, 17 (1995), pp. 171-179

17E. Regev, J. Lustmann, R. NashefAtraumatic teeth extraction in bisphosphonate-treated patients J Oral Maxillofac Surg, 66 (2008), pp. 1157-1161

18J.D. SpougeHemostasis in dentistry, with special reference to hemocoagulation. II. Principles underlying clinical hemostatic practices in normal patientsOral Surg Oral Med Oral Pathol, 18 (1964), pp. 583-592

19F. Amato, A.D. Mirabella, U. Macca, D.P. TarnowImplant site development by orthodontic forced extraction: a preliminary studyInt J Oral Maxillofac Implants, 27 (2012), pp. 411-420

20M. Korayem, C. Flores-Mir, U. Nassar, K. OlfertImplant site development by orthodontic extrusion. a systematic reviewAngle Orthod, 78 (2008), pp. 752-760

21T. Mantzikos, I. ShamusForced eruption and implant site development: an osteophysiologic response Am J Orthod Dentofacial Orthop, 115 (1999), pp. 583-591

22T. Mantzikos, I. ShamusForced eruption and implant site development: soft tissue response Am J Orthod Dentofacial Orthop, 112 (1997), pp. 596-606

$23 \mathrm{H}$. Salama, M. SalamaThe role of orthodontic extrusive remodeling in the enhancement of soft and hard tissue profiles prior to implant placement: a systematic approach to the management of extraction site defectsInt J Periodontics Restorative Dent, 13 (1993), pp. 312-333

24B.U. Zachrisson, M. Rosa, S. ToreskogCongenitally missing maxillary lateral incisors: canine substitution [Point/Couinterpoint]Am J Orthod Dentofacial Orthop, 139 (2011), p. 434

25J.H. Park, S. Okadakage, Y. Sato, Y. Akamatsu, K. TaiOrthodontic treatment of a congenitally missing maxillary lateral incisorJ Esthet Restor Dent, 22 (2010), pp. 297-312 
26E.M. Czochrowska, A.B. Skaare, A. Stenvik, B.U. ZachrissonOutcome of orthodontic space closure with a missing maxillary central incisorAm J Orthod Dentofacial Orthop, 123 (2003), pp. 597-603

$27 \mathrm{~S}$ Robertsson, B. MohlinThe congenitally missing upper lateral incisor. A retrospective study of orthodontic space closure versus restorative treatmentEur J Orthod, 22 (2000), pp. 697-710 28R. SabriManagement of missing maxillary lateral incisorsJ Am Dent Assoc, 130 (1999), pp. 80-84

29R.J. Cronin Jr., L.J. Oesterle, D.M. RanlyMandibular implants and the growing patientInt J Oral Maxillofac Implants, 9 (1994), pp. 55-62

30G.G. Nordquist, R.W. McNeillOrthodontic vs. restorative treatment of the congenitally absent lateral incisor-long term periodontal and occlusal evaluationJ Periodontol, 46 (1975), pp. 139-143

31G.S. Silveira, N.V. de Almeida, D.M. Pereira, C.T. Mattos, J.N. MuchaProsthetic replacement vs space closure for maxillary lateral incisor agenesis: a systematic reviewAm J Orthod Dentofacial Orthop, 150 (2016), pp. 228-237

32A.H. Muhamad, W. Nezar, A. AzzaldeenManaging congenitally missing lateral incisors with single tooth implantsDent Oral Craniofac Res, 2 (2016), pp. 318-324

33A. Jamilian, L. Perillo, M. RosaMissing upper incisors: a retrospective study of orthodontic space closure versus implantProg Orthod, 16 (2015), p. 2

34M. Rosa, B.U. ZachrissonThe space-closure alternative for missing maxillary lateral incisors: an update J Clin Orthod, 44 (2010), pp. 540-549

35B.U. ZachrissonSingle implant-supported crowns in the anterior maxilla-potential esthetic long-term (>5 years) problemsWorld J Orthod, 7 (2006), pp. 306-312

36B. Thilander, J. Odman, U. LekholmOrthodontic aspects of the use of oral implants in adolescents: a 10-year follow-up studyEur J Orthod, 23 (2001), pp. 715-731

37U. GrunderStability of the mucosal topography around single-tooth implants and adjacent teeth: 1year resultsInt J Periodontics Restorative Dent, 20 (2000), pp. 11-17

38T. JemtMeasurements of tooth movements in relation to single-implant restorations during 16 years: a case reportClin Implant Dent Relat Res, 7 (2005), pp. 200-208

39J.P. Bernard, J.P. Schatz, P. Christou, U. Belser, S. KiliaridisLong-term vertical changes of the anterior maxillary teeth adjacent to single implants in young and mature adults. A retrospective studyJ Clin Periodontol, 31 (2004), pp. 1024-1028

40R.E. Jung, B.E. Pjetursson, R. Glauser, A. Zembic, M. Zwahlen, N.P. LangA systematic review of the 5year survival and complication rates of implant-supported single crownsClin Oral Implants Res, 19 (2008), pp. 119-130 
41M.S. Scurria, J.D. Bader, D.A. ShugarsMeta-analysis of fixed partial denture survival: prostheses and abutmentsJ Prosthet Dent, 79 (1998), pp. 459-464

42M. Hof, B. Pommer, G.D. Strbac, D. Sütö, G. Watzek, W. ZechnerEsthetic evaluation of single-tooth implants in the anterior maxilla following autologous bone augmentationClin Oral Implants Res, 24 (2013), pp. 88-93

43B.U. ZachrissonLong-term experience with direct-bonded retainers: update and clinical advice J Clin Orthod, 41 (2007), pp. 728-737 